Article

\title{
Dual-Zone Active Noise Control Algorithm
}

\author{
Ran Wang ${ }^{1,2}$, Xiaolin Wang ${ }^{1, *}$, Jingwei Liu ${ }^{1,2}$ and Jun Yang ${ }^{1}$ \\ 1 Key Laboratory of Noise and Vibration Research, Institute of Acoustics, Chinese Academy of Sciences, \\ No. 21 North 4th Ring Road, Haidian District, Beijing 100190, China; wangran@mail.ioa.ac.cn (R.W.); \\ liujingwei@mail.ioa.ac.cn (J.L.); jyang@mail.ioa.ac.cn (J.Y.) \\ 2 School of Electronic, Electrical and Communication Engineering, University of Chinese Academy of Sciences, \\ No. 19(A) Yuquan Road, Shijingshan District, Beijing 100049, China \\ * Correspondence: wangxiaolin@mail.ioa.ac.cn
}

Received: 3 November 2019; Accepted: 16 December 2019; Published: 18 December 2019

Featured Application: The algorithms proposed in this paper can be applied in directional ANC where quiet zone is formed with small increase of sound power outside the quiet zone. Moreover, the algorithms also can be applied in multi-zone ANC research.

\begin{abstract}
When active noise control (ANC) is applied to acquire a 'quiet zone', it may produce an increase in the sound power outside the quiet zone and a change in the primary sound field, which are undesirable in anti-detection and personal audio. To obtain a large noise reduction in the control zone and a small increase of sound power outside the control zone, three wideband ANC algorithms are proposed based on the acoustic contrast control (ACC), least-squares (LS), and least-squares with acoustic contrast control (SFR-ACC) algorithms. With a loudspeaker array as the secondary source, dual-zone ANC with directivity, which realizes noise reduction in one zone without changing the sound power in the other zone, is achieved. Compared with the traditional LS algorithm, the three algorithms proposed in this paper can not only realize that the sound power outside the control zone is increased by less than $1 \mathrm{~dB}$, but also reduce the noise in the control zone by more than $10 \mathrm{~dB}$, which provides a new solution to multi-zone ANC research.
\end{abstract}

Keywords: local active noise control; LS algorithm; ACC algorithm

\section{Introduction}

Active noise control (ANC) is widely used as it reduces noise at low frequencies. Global ANC can achieve a larger-space noise reduction in the control zone [1-3], while local ANC can realize multi-zone noise reduction [4-6]. However, under some special circumstances, such as anti-detection and noise reduction between seats where the sound field is distributed over multiple zones, it is necessary to decrease the increase of sound power outside the control zone when a large noise reduction is required in the control zone to prevent the detection and disturbance of others. In other words, control with directivity is needed. In addition, most of the existing control algorithms focus on how to maximize the noise reduction in the control zone only, and neglect the increase of sound power outside the control zone. Therefore, it is necessary to apply a new control algorithm to consider both of the two parts.

While there is scarce research in ANC, enormous effective algorithms are proposed to form a directional output from a loudspeaker array for sound field reproduction [7-9]. Elliott et al. applied the acoustic contrast control (ACC) [10] algorithm to an active headrest [11]. For active headrest systems, the ACC algorithm eliminates the effect between the adjacent seats and increases the noise reduction in the control zone by $20 \mathrm{~dB}$. Liu et al. [12] used a constrained least-squares (CLS) algorithm to make the output of the loudspeaker array directional, thereby, achieving directed noise reduction. In addition, Liu [13] proposed a control strategy that combines an optimized loudspeaker array and zero pressure 
constrained points, in order to control the scattered sound field of a rigid sphere. This new control algorithm has good reduction performance in the target areas and less amplification outside the target zone. However, this new control algorithm is not a wideband. In addition, the least-squares approach with acoustic contrast control constraint (SFR-ACC) [14] balancing the spatial average error and the acoustic contrast has been used in personal audio system. However, this algorithm has never been applied in ANC.

To verify the feasibility of the SFR-ACC algorithm in ANC and to realize multi-zone directional ANC, three wideband ANC algorithms based on the ACC, CLS and SFR-ACC concepts are proposed in this paper. Using a loudspeaker array, the three algorithms form directional outputs and realize dual-zone ANC by reducing the noise in one zone, without changing the sound power in the other zone. The three methods described in this paper utilize white noise to model a wideband transfer function that reduces time and resource consumption and makes implementation easier. Compared with the traditional LS algorithm in dual-zone ANC, the three algorithms proposed in this paper, not only realize that the sound power in one zone is increased by less than $1 \mathrm{~dB}$, but also reduce the noise in the other zone by more than $10 \mathrm{~dB}$. The remainder of this paper is organized as follows. In Section 2, a brief overview of the dual-zone ANC, the mathematical formulation of the three algorithms, and the criteria of the algorithm performance are outlined. The experimental results are provided in Section 3. Finally, some conclusions are given in Section 4.

\section{Dual-Zone Active Noise Control}

The geometry of the dual-zone ANC system is shown in Figure 1. One zone is defined as the control zone and the other zone is defined as the noncontrol zone. The control zone is the quiet zone and the noncontrol zone's sound power remains unchanged when ANC is on. Therefore, the dual-zone ANC system in this paper is not aimed at realizing ANC in both zones but at realizing a directional ANC. The primary source is located on the negative half of the $y$-axis. The secondary source array consisting of $N_{s}$ loudspeakers is evenly distributed on the $x$-axis. The control zone and the noncontrol zone have $N_{e}$ and $N_{c}$ uniformly distributed microphones, respectively, and are symmetrically distributed in the XOY plane. The distance between the primary source and the origin is $L_{p s}$. The length of the loudspeaker array is $L_{s}$ with spacing $d_{s s}$. The distance between the center of the control points and the origin is $R_{e c}$, and the distance between the control points is $d_{\mathrm{e}}$. The noncontrol points are identical in configuration to the control points with symmetric coordinate points on the $y$-axis. The control zone and the noncontrol zone are located in the $-\theta$, and $\theta$ directions, respectively, relative to the center.

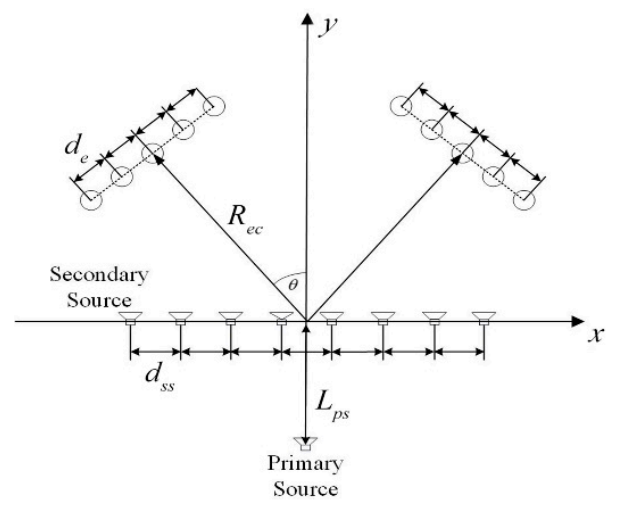

Figure 1. The geometry of the dual-zone active noise control (ANC) system.

The block diagram of the dual-zone ANC system is shown in Figure 2. When the primary source and the secondary source are working together, the broadband sound pressure at the control points can be represented as,

$$
\mathbf{P}_{e t}=\mathbf{P}_{e p}+\mathbf{P}_{e s}=\mathbf{G}_{p e} q_{p}+\mathbf{G}_{s e} q_{s}
$$


and the sound pressure at the noncontrol points can be written as,

$$
\mathbf{P}_{c t}=\mathbf{P}_{c p}+\mathbf{P}_{c s}=\mathbf{G}_{p c} q_{p}+\mathbf{G}_{s c} q_{s}
$$

where $\mathbf{P}_{e p}$ and $\mathbf{P}_{c p}$ are the sound pressure of the control points and the noncontrol points produced by the primary source, respectively. $\mathbf{P}_{e p}$ is an $N_{e} \times L$ vector, and $\mathbf{P}_{c p}$ is an $N_{c} \times L$ vector, where $L$ is the number of frequencies. $\mathbf{P}_{e s}$ and $\mathbf{P}_{\mathcal{~}}$ are the sound pressure of the control points and noncontrol points produced by the secondary source, respectively. $\mathbf{P}_{e s}$ is an $N_{e} \times L$ vector, and $\mathbf{P}_{c s}$ is an $N_{c} \times L$ vector. $\mathbf{G}_{p e}$ and $\mathbf{G}_{p c}$ are the transfer functions between the primary source and the control points and noncontrol points, respectively. $\mathbf{G}_{p e}$ is an $\left(N_{e} \times 1\right) \times L$ matrix, and $\mathbf{G}_{p c}$ is an $\left(N_{c} \times 1\right) \times L$ matrix. $\mathbf{G}_{s e}$ and $\mathbf{G}_{s c}$ are the transfer functions between the secondary source and the control points and noncontrol points, respectively. $\mathbf{G}_{s e}$ is an $\left(N_{e} \times N_{s}\right) \times L$ matrix, and $\mathbf{G}_{s c}$ is an $\left(N_{c} \times N_{s}\right) \times L$ matrix. In addition, $q_{p}$ and $q_{s}$ are the strength of the primary source and the secondary source, respectively.

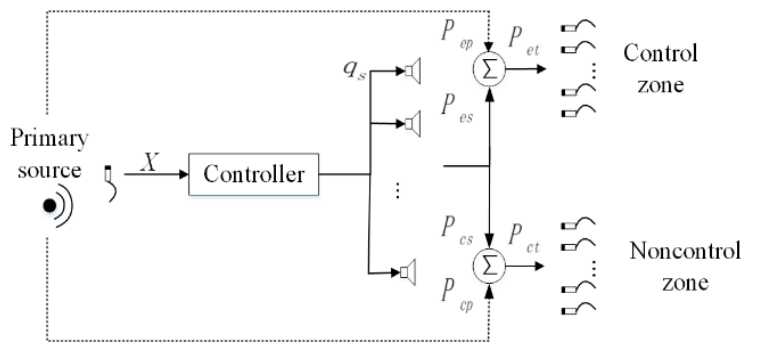

Figure 2. Block diagram of the dual-zone ANC system.

The key concept of the dual-zone ANC is how to solve the strength of the secondary source. There are two targets: One is to minimize the total energy in the control zone, corresponding to noise reduction; the other is to minimize the input energy to the noncontrol zone produced by the secondary sources, corresponding to forming directional outputs.

When the constraint LS algorithm is applied to dual-zone ANC, the total pressure of the control zone can be represented as in (1). However, the total pressure of the noncontrol zone is expressed as,

$$
\mathbf{P}_{c t}=\mathbf{P}_{c p}+\mathbf{P}_{c s}=\mathbf{G}_{p c} q_{p}
$$

where the response of the secondary loudspeaker array at the noncontrol zone is constrained to zero. Therefore, the cost function of the dual-zone ANC can be written as,

$$
\mathbf{J}_{\mathrm{CLS}}=\left(\left[\begin{array}{c}
\mathbf{G}_{s e} \\
\mathbf{G}_{s c}
\end{array}\right] q_{s}+\left[\begin{array}{c}
\mathbf{G}_{p e} \\
0
\end{array}\right] q_{p}\right)^{\mathrm{H}}\left(\left[\begin{array}{l}
\mathbf{G}_{s e} \\
\mathbf{G}_{s c}
\end{array}\right] q_{s}+\left[\begin{array}{c}
\mathbf{G}_{p e} \\
0
\end{array}\right] q_{p}\right)
$$

Then, the least square solution to Equation (4) can be written as,

$$
\mathbf{q}_{s}{ }^{C L S}=-\left(\left[\begin{array}{l}
\mathbf{G}_{s e} \\
\mathbf{G}_{s c}
\end{array}\right]^{H}\left[\begin{array}{l}
\mathbf{G}_{s e} \\
\mathbf{G}_{s c}
\end{array}\right]+\lambda \mathbf{I}\right)^{-1}\left[\begin{array}{c}
\mathbf{G}_{s e} \\
\mathbf{G}_{s c}
\end{array}\right]^{H}\left[\begin{array}{c}
\mathbf{G}_{p e} \\
0
\end{array}\right] q_{p}
$$

where $\mathbf{I}$ is an identity matrix and $\lambda$ is the regularization parameter. Equation (5) is the secondary source strength when the CLS algorithm is applied to dual-zone ANC.

The cost function of the ACC algorithm does not contribute to noise reduction when it is applied to dual-zone ANC unless it is equalized $[14,15]$. The optimal strength of the secondary source when it is applied in dual-zone ANC is given as,

$$
\mathbf{q}_{s}{ }^{A C C}=\frac{\operatorname{abs}\left\{\mathbf{w}_{\text {opt }}{ }^{H} \mathbf{G}_{s e}{ }^{H} \mathbf{P}_{e p}\right\}}{\mathbf{w}_{\text {opt }}{ }^{H}\left(\mathbf{G}_{s e}{ }^{H} \mathbf{G}_{s e}\right) \mathbf{w}_{\text {opt }}} \exp \left(j \angle\left\{\mathbf{w}_{\text {opt }}{ }^{\mathrm{H}} \mathbf{G}_{s e}{ }^{H} \mathbf{P}_{e p}\right\}\right) \mathbf{w}_{\text {opt }}
$$


where $\mathbf{w}_{\text {opt }}$ equals the unit eigenvector, which corresponds to the largest eigenvalue of the matrix $\left(\mathbf{G}_{s c}^{H} \mathbf{G}_{s c}+\lambda \mathbf{I}\right)^{-1}\left(\mathbf{G}_{s e}^{H} \mathbf{G}_{s e}\right)$. Additionally, abs $\{\cdot\}$ and $\angle\{\cdot\}$ represent the modulus, and argument of the elements, respectively.

When SFR-ACC is applied to dual-zone ANC, the cost function can be rewritten as,

$$
\begin{gathered}
\min \left\|\mathbf{G}_{s e} \mathbf{q}_{s}+\mathbf{P}_{e p}\right\|_{2}^{2} \\
\text { s.t. } 10 \log C \geq 10 \log C_{s t}
\end{gathered}
$$

where $C$ denotes the acoustic contrast, which can be defined as the ratio of acoustic potential energy between the control zone and the noncontrol zone. $C_{s t}$ is a constant that is smaller than $C_{\max }$, and $C_{\max }$ is the largest eigenvalue of the matrix $\left(\mathbf{G}_{s c}^{H} \mathbf{G}_{s c}+\lambda \mathbf{I}\right)^{-1}\left(\mathbf{G}_{s e}^{H} \mathbf{G}_{s e}\right)$. Because this function is a non-convex problem, it has to be transferred into a convex problem [13] and then, can be solved by the CVX package [16].

Once the strength of the secondary source is obtained by Equations (5)-(7), the filter coefficient $\mathbf{q}_{s}{ }^{\prime}$ can be written as,

$$
\mathbf{q}_{s}^{\prime}=\frac{\mathbf{q}_{s}}{\mathbf{X}}
$$

where $\mathbf{X}$ is the reference signal of $L$ frequencies. Therefore, the wideband control filter coefficients of the dual-zone ANC systems are obtained in Equation (8), based on different algorithms.

Unlike sound field reproduction, evaluating the performance of the three algorithms in dual-zone ANC requires redefining new criteria. The first criterion refers to the noise reduction in the control zone, which can be expressed as,

$$
\boldsymbol{R e}=10 \log \left(\frac{\left\|\mathbf{P}_{e t}\right\|_{2}^{2}}{\left\|\mathbf{P}_{e p}\right\|_{2}^{2}}\right)
$$

and stands for the noise reduction performance of the secondary loudspeaker array in the control zone.

The second criterion refers to the increase of sound power in the noncontrol zone, which is defined as,

$$
\text { Isp }=a b s\left(10 \log \left(\frac{\left\|\mathbf{P}_{c t}\right\|_{2}^{2}}{\left\|\mathbf{P}_{c p}\right\|_{2}^{2}}\right)\right)
$$

which is the abbreviation of the increase of sound power and stands for the energy change in the sound field, produced by the primary source, due to the presence of the secondary loudspeaker array. The smaller the value of Isp, the better the performance of the algorithm.

\section{Experimental Results}

The dual-zone ANC experiments are carried out in an anechoic chamber (Figure 3a). The TMS320C6678 DSP is utilized as the controller and a feedforward control is realized. The secondary loudspeaker array consists of eight speaker units spaced $0.12 \mathrm{~m}$ apart (Figure $3 \mathrm{~b}$ ). There are 11 microphones: a reference microphone, five control points and five noncontrol points. The spacing between the control points and the noncontrol points is $0.08 \mathrm{~m}$. The center of the secondary loudspeaker array is taken as the origin. The distance between the center of the control points and the origin is $1.5 \mathrm{~m}$. The noncontrol points have the same configuration as the control points. The control points and noncontrol points are located in the $-30^{\circ}$ and $30^{\circ}$ directions deviated from the center of the secondary loudspeaker array, respectively. The primary source is placed $1.5 \mathrm{~m}$ from the origin. The sampling frequency of the dual-zone ANC system is $8000 \mathrm{~Hz}$. The loudspeakers are positioned on the same horizontal plane. 


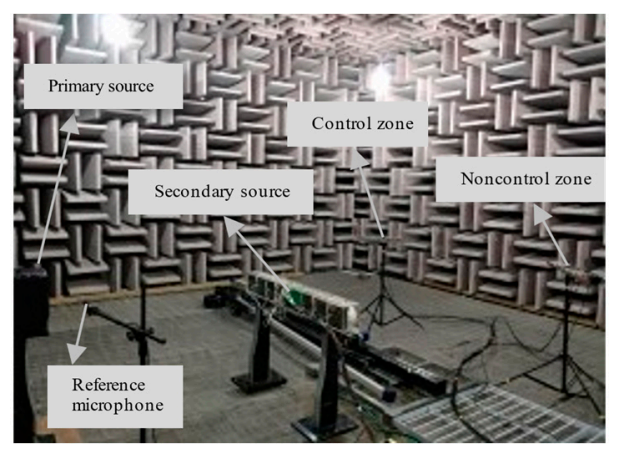

(a)

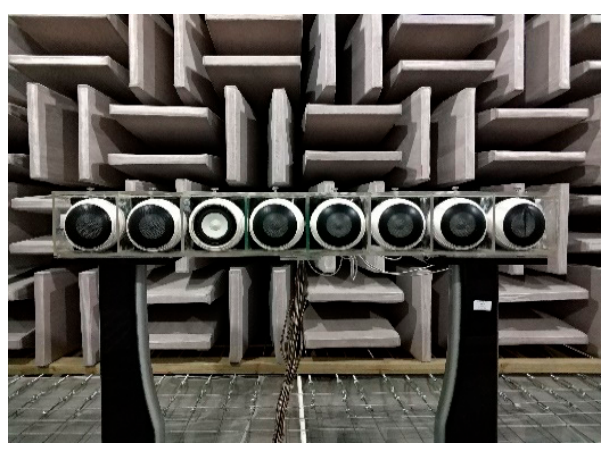

(b)

Figure 3. The dual-zone ANC system and the secondary loudspeaker array: (a) is the dual-zone ANC system; and (b) is the secondary loudspeaker array.

The transfer function between the secondary loudspeaker array and the control points and the noncontrol points are initially measured with white noise. Then, a white noise signal is emitted by the primary source, which can be obtained by the reference microphone as a reference signal $\mathbf{X}$. The algorithms proposed in this paper and the reference signal $\mathbf{X}$ are used to obtain the wideband filter coefficient in the frequency domain. Then, the time domain filter coefficient can be acquired by inverse Fourier transformation. The frequency range of interest is from 300 to $1200 \mathrm{~Hz}$. The time domain experimental results measured at discrete frequencies with $50 \mathrm{~Hz}$ spacing of the proposed three algorithms are shown in Figure 4 when $\lambda=10^{-4}$.

Figure $4 \mathrm{a}, \mathrm{b}$ shows that the algorithms proposed in this paper have a good wideband performance. From 300 to $1200 \mathrm{~Hz}$, the average noise reduction in the control point is more than $10 \mathrm{~dB}$ and the average sound power increase is less than $1 \mathrm{~dB}$. From Figure $4 \mathrm{~b}$, the increase of sound power of LS algorithms is much larger than that of the algorithms proposed in this paper, which verifies that the three wideband algorithms proposed in this paper can realize directional ANC, where the secondary loudspeaker array processes only noise reduction in the control zone, without changing the initial sound field of the noncontrol zone.

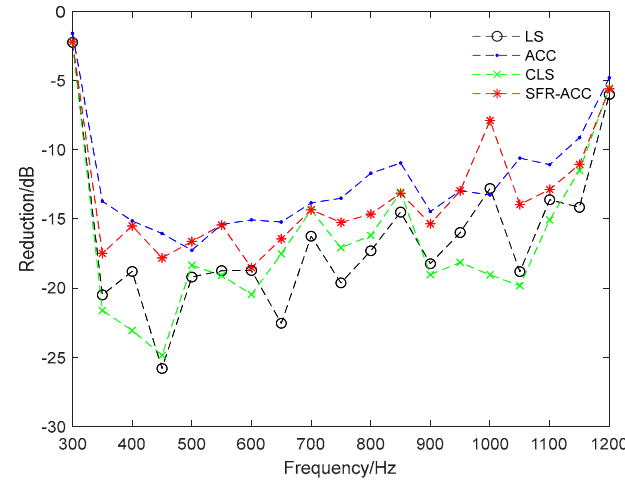

(a)

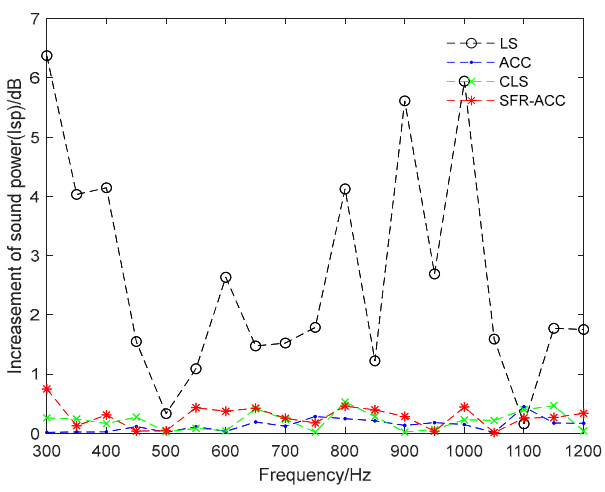

(b)

Figure 4. The experimental results of the dual-zone ANC from 300 to $1200 \mathrm{~Hz}$ : (a) is the results of reduction (Re) in the control zone; and (b) is the increase of sound power (Isp) in the noncontrol zone.

Although, the algorithm based on ACC has a smaller noise reduction in the control zone, it has better directivity. Therefore, the increase of sound power in the noncontrol zone is the smallest of the three proposed algorithms. The algorithm based on CLS has better noise reduction. At $900 \mathrm{~Hz}$, the noise reduction difference between LS and the CLS is small, which means that the constraint to the LS does not affect the robustness of the LS algorithm. In addition, the experimental results of the proposed algorithm, based on SFR-ACC, can also realize dual-zone ANC with directivity, which verifies its feasibility in ANC systems. From the noise reduction curve in Figure 4a, the trend of the 
SFR-ACC algorithm is the same as the results of the LS algorithm. However, because this algorithm combines the LS algorithm and the ACC algorithm, the robustness of SFR-ACC is determined by both of them. Therefore, the noise reduction level is smaller than in the LS algorithm.

In addition, the small noise reduction at $300-400 \mathrm{~Hz}$ and $1100-1200 \mathrm{~Hz}$ is caused by data truncation when the inverse Fourier transform is utilized. To avoid this phenomenon, the frequency range should be expanded to ensure that the frequency of interest is within the measured frequency, when modeling the transfer function and calculating the filter coefficients.

The theoretical results are the off-line simulation results based on measured data. Figure $5 \mathrm{a}, \mathrm{b}$ shows that the theoretical results are in good agreement with the experimental results. However, the experimental noise reduction in the proposed SFR-ACC algorithm is smaller than the theoretical noise reduction, for two reasons. On one hand, the robustness of this algorithm is determined by both the ACC algorithm and the LS algorithm, which leads to sensitivity to environmental disturbances. On the other hand, the consistency of the secondary loudspeaker array is poor.

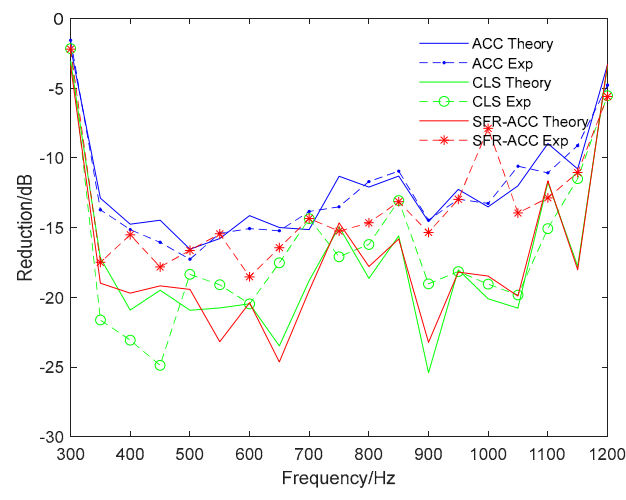

(a)

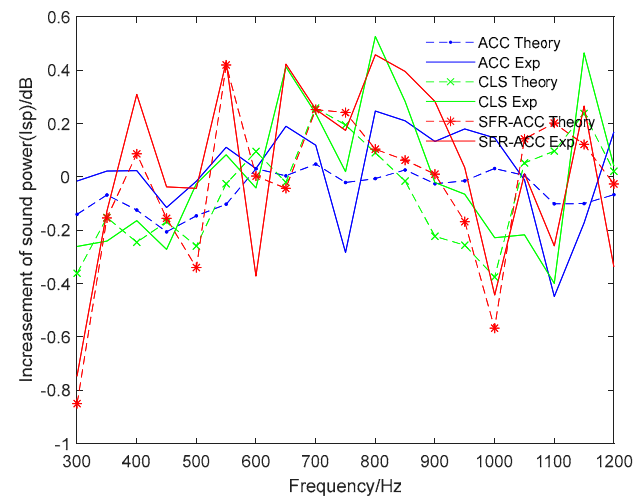

(b)

Figure 5. The contrasting results of the dual-zone ANC from 300 to $1200 \mathrm{~Hz}$ based on the algorithms proposed in this paper: (a) is the contrasting results of the theoretical and experimental reduction (Re); and (b) is the contrasting results of the theoretical and experimental increase of sound power (Isp).

\section{Conclusions and Discussion}

To realize noise reduction in one zone without changing the sound pressure in the other zone, three wideband ANC algorithms, based on the ACC, CLS, and SFR-ACC concepts are proposed in this paper. With a loudspeaker array and the white noise modeling method, dual-zone ANC with directivity is realized. Compared with the LS algorithm, the proposed three methods can reduce the noise in the control zone, as well as reduce the increase of sound power in the noncontrol zone. From 300 to $1200 \mathrm{~Hz}$, the noise reduction in the control zone is more than $10 \mathrm{~dB}$, and the increase of sound power in the noncontrol zone is less than $1 \mathrm{~dB}$. Compared with the experimental results, the off-line simulation results (theoretical results) have a good agreement, which verifies that the algorithms proposed in this paper are effective and efficient. In addition, to acquire better noise reduction, the frequency range of modeling and calculating filter coefficients should be much wider than the frequency of interest.

Considering the dual-zone ANC system proposed in this paper as one single unit, and assuming that one control system consists of many units, an arbitrary quiet zone and noncontrol zone can be obtained in space. Therefore, the distributed ANC can also be realized based on the algorithm and the ANC system proposed in this paper. Moreover, to acquire a robust ANC system, the method for choosing the regularization parameters is a valuable research issue which will be discussed in future work.

Author Contributions: Conceptualization, R.W., J.L., and X.W.; writing-original draft preparation, R.W.; writing-review and editing, X.W. and J.Y.; investigation, R.W.; supervision, X.W. and J.Y. All authors have read and agreed to the published version of the manuscript. 
Funding: This research was supported by National Major Projects (No. 613427).

Conflicts of Interest: The authors declare no conflict of interest.

\section{References}

1. Kajikawa, Y.; Gan, W.S.; Kuo, S.M. Recent advances on active noise control: Open issues and innovative applications. Apsipa Trans. Signal Inf. Process. 2012, 1, e3. [CrossRef]

2. Guo, J.; Pan, J.; Bao, C. Actively created quiet zones by multiple control source in free space. J. Acoust. Soc. Am. 1997, 101, 1492-1501. [CrossRef]

3. Nelson, P.A.; Elliott, S.J. Active control of sound. Phys. Today 1993, 46, 75-76. [CrossRef]

4. Kuo, S.M.; Morgan, D.R. Active noise control: A tutorial review. Proc. IEEE 1999, 87, 943-973. [CrossRef]

5. Tseng, W.K. Local active noise control using a novel method of designing quiet zones. Control Eng. Pract. 2011, 19, 1450-1458. [CrossRef]

6. Boulandet, R.; Laurence, T.; Hervé, L. Design of Remote Quiet Zones Using Spot-Type Sound Reducers. Acta Acust. United Acust. 2017, 103, 858-868. [CrossRef]

7. Jones, M.; Elliott, S.J. Personal audio with multiple dark zones. J. Coustical Soc. Am. 2008, 124, 3497-3506. [CrossRef] [PubMed]

8. Simon Galvez, M.F.; Elliott, S.J.; Cheer, J. A superdirective array of phase shift sources. J. Acoust. Soc. Am. 2012, 132, 746-756. [CrossRef] [PubMed]

9. Cheer, J.; Elliott, S.J.; Kim, Y.; Choi, J.W. Practical implementation of personal audio in a mobile device. J. Audio Eng. Soc. 2013, 61, 290-300.

10. Choi, J.W.; Kim, Y.H. Generation of an acoustically bright zone with an illuminated region using multiple sources. J. Acoust. Soc. Am. 2002, 111, 1695-1700. [CrossRef] [PubMed]

11. Elliott, S.J.; Jones, M. An active headrest for personal audio. J. Acoust. Soc. Am. 2006, 119, $2702-2709$. [CrossRef] [PubMed]

12. Liu, J.; Xiaolin, W.; Ming, W.; Jun, Y. Actively created quiet zones using directional array of loudspeakers. In Proceedings of the 24th International Congress on Sound and Vibration, London, UK, 23-27 July 2017.

13. Liu, J.; Xiaolin, W.; Wu, M.; Jun, Y. An active control strategy for the scattered sound field control of a rigid sphere. J. Acoust. Soc. Am. 2018, 144, EL52-EL58. [CrossRef] [PubMed]

14. Yefeng, C.; Ming, W.; Jun, Y. Sound reproduction in personal audio systems using the least-squares approach with acoustic contrast control constraint. J. Acoust. Soc. Am. 2014, 135, 734-741.

15. Choi, J.W.; Kim, Y.H. Active control for the enhancement of sound field. In Proceedings of the Inter-noise \& Noise-control Congress Conference, Daejeon, Korea, 22-25 August 2004.

16. CVX: Matlab Software for Disciplined Convex Programming, Version 2.0 Beta. Available online: http: //cvxr.com/cvx (accessed on 15 December 2018).

(C) 2019 by the authors. Licensee MDPI, Basel, Switzerland. This article is an open access article distributed under the terms and conditions of the Creative Commons Attribution (CC BY) license (http://creativecommons.org/licenses/by/4.0/). 